\title{
Thermodynamic Properties of Poly(ethylene glycol) and Poly(tetrahydrofuran) \\ II. Conformational Entropy of Melting
}

\author{
Yoshiharu Tsujita, Takuhei Nose, and Toshio Hata \\ Department of Polymer Chemistry, Tokyo Institute of Technology, \\ Ookayama 2-12-1, Meguro-ku, Tokyo, Japan.
}

(Received June 20, 1973)

\begin{abstract}
The entropy of melting $\Delta S_{\mathrm{m}}$ was studied for poly(ethylene glycol) (PEG) and poly(tetrahydrofuran) (PTHF). The $\Delta S_{\mathrm{m}}$ was divided into three parts: $\Delta S_{\mathrm{dis}}$, the entropy change due to disordering of the molecular arrangement at constant volume; $\Delta S_{\mathrm{c}}$, the one due to the change in chain conformation; and $\Delta S_{\mathrm{V}}$, the entropy arising from the change in volume. $\Delta S_{\mathrm{V}}$ was evaluated from the experimental $P-V-T$ relations for these polymers, with the volume dependence of $(\partial S / \partial V)_{T}=(\partial P / \partial T)_{V}$ taken into consideration; $\Delta S_{\text {dis }}$ was estimated with the aid of thermodynamic data for simple spherical molecules. The resulting conformational entropies of melting per mole of bonds were $1.50 \mathrm{cal} / \mathrm{deg}$ for PEG with molecular weight $M_{n}=605,1.41 \mathrm{cal} / \mathrm{deg}$ for PEG with $M_{n}=$ $1020,1.40 \mathrm{cal} / \mathrm{deg}$ for PEG with $M_{n}=7500$, and $1.53 \mathrm{cal} / \mathrm{deg}$ for PTHF with $M=40000$.

KEY WORDS Entropy of Melting / Conformational Entropy /

Clausius-Clapeyron Equation / Thermal Pressure Coefficient /

Pressure / Melting Point / Poly(ethylene glycol) / Poly(tetrahydrofuran) /
\end{abstract}

One of the most characteristic aspects of polymer melting is the change in chain conformation which occurs on melting. In this paper, in order to further our understanding of this effect on the thermodynamics of melting of crystalline polymers, the entropies of melting for poly(ethylene glycol)s (PEG) and poly(tetrahydrofuran) (PTHF) were estimated by the same method as in a preceding paper on polyethylene and eicosane. ${ }^{1}$

The entropy of melting $\Delta S_{\mathrm{m}}$ may be separated into three parts as has been proposed in a previous paper. $^{1}$ They are the disordering entropy $\Delta S_{\mathrm{dis}}$, the conformational entropy $\Delta S_{\mathrm{c}}$, and the entropy of melting due to volume change $\Delta S_{\nabla}$. From the data for pressurevolume-temperature $(P-V-T)$ relations these three parts can be estimated. The entropy change $\Delta S_{\nabla}$ is evaluated by application of the Maxwell relation $(\partial S / \partial V)_{T}=(\partial P / \partial T)_{V}$. In this case, it is important to take the volume dependence of $(\partial P / \partial T)_{V}$ into account, although Starkweather and Boyd ${ }^{2}$ treated $(\partial P / \partial T)_{V}$ as constant. The disordering entropy $\Delta S_{\mathrm{dis}}$ is ob- tained from the entropy changes of disordering for $\mathrm{Ar}$ and $\mathrm{CCl}_{4}$. Finally, the conformational entropy $\Delta S_{\mathrm{c}}$ is determined by subtracting these two parts from $\Delta S_{\mathrm{m}}$. The values of $\Delta S_{\mathrm{c}}$ thus obtained for PEG of different molecular weights and that for PTHF are compared with those of polyethylene and eicosane.

\section{EXPERIMENTAL}

\section{Materials and Experimental Methods}

Three samples of poly(ethylene glycol) with different molecular weight were provided by Nippon Oil and Fats Company, Ltd. These were the same as those used in a previous paper. ${ }^{3}$ They are designated PEG 600, PEG 1000 , and PEG 6000. Their molecular weights $M$ are indicated in Table I. It was shown that these had narrow distributions of molecular weights. The sample of poly(tetrahydrofuran) used also had a narrow distribution of molecular weights $(M=40000) .^{3}$ It was a gift from Dr. T. Fujimoto of Nagoya University. The samples of PEG were crystallized by slow cool- 
Table I. Thermodynamic quantities of melting

\begin{tabular}{lccccc}
\hline & & PEG 600 & PEG 1000 & PEG 6000 & PTHF \\
\hline$M$ & & 605 & 1020 & 7500 & 40000 \\
$T_{\mathrm{m}}$ & ${ }^{\circ} \mathrm{C}$ & 24.6 & 41.1 & 66.0 & 42.7 \\
$\mathrm{~d} T_{\mathrm{m}} / \mathrm{d} P$ & $\mathrm{deg} / \mathrm{kg} \mathrm{cm}$ & 0.0123 & 0.0145 & 0.0154 & 0.0181 \\
$\Delta V_{\mathrm{m}}$ & $\mathrm{cc} / \mathrm{g}$ & $0.080_{1}$ & 0.0899 & $0.095_{2}$ & 0.199 \\
$\Delta S_{\mathrm{m}}$ & $\mathrm{cal} / \mathrm{mol} \mathrm{deg}$ & 6.71 & 6.40 & 6.38 & 11.14 \\
$\Delta H_{\mathrm{m}}$ & $\mathrm{kcal} / \mathrm{mol}$ & 2.00 & 2.01 & 2.16 & 3.52 \\
$\Delta S_{\mathrm{V}}$ & $\mathrm{cal} / \mathrm{mol} \mathrm{deg}$ & 1.71 & 1.66 & 1.68 & 3.00 \\
$\left(\Delta S_{\mathrm{m}}\right)_{V}$ & $\mathrm{cal} / \mathrm{mol} \mathrm{deg}$ & 5.00 & 4.74 & 4.70 & 8.14 \\
$\Delta S_{\mathrm{dis}}$ & $\mathrm{cal} / \mathrm{mol} \mathrm{deg}$ & 0.49 & 0.50 & 0.50 & 0.75 \\
$\Delta S_{\mathrm{c}}$ & $\mathrm{cal} / \mathrm{mol} \mathrm{deg}$ & 4.51 & 4.24 & 4.20 & 7.39 \\
$\Delta S_{\mathrm{c}} /$ bond & $\mathrm{cal} / \mathrm{mol} \mathrm{deg}$ & 1.50 & 1.41 & 1.40 & 1.48 \\
\hline
\end{tabular}

ing from the molten state; the sample of PTHF was crystallized at room temperature. The crystallinities determined by the density method were $77.9,72.4,85.0$, and $61.4 \%$ for PEG 600, PEG 1000, PEG 6000, and PTHF, respectively. The volume change on melting, $\Delta V_{\mathrm{m}}$, the pressure dependence of the melting temperature, $\mathrm{d} T_{\mathrm{m}} / \mathrm{d} P$, and the thermal pressure coefficient were derived from the $P-V-T$ relations. The apparatus and procedures used for the measurements of the $P-V-T$ relations have been described elsewhere. ${ }^{4}$

\section{Experimental Results}

Volumes of the solid state at the melting temperature $T_{\mathrm{m}}$ were estimated by extrapolation of the data at temperatures lower than the pre-

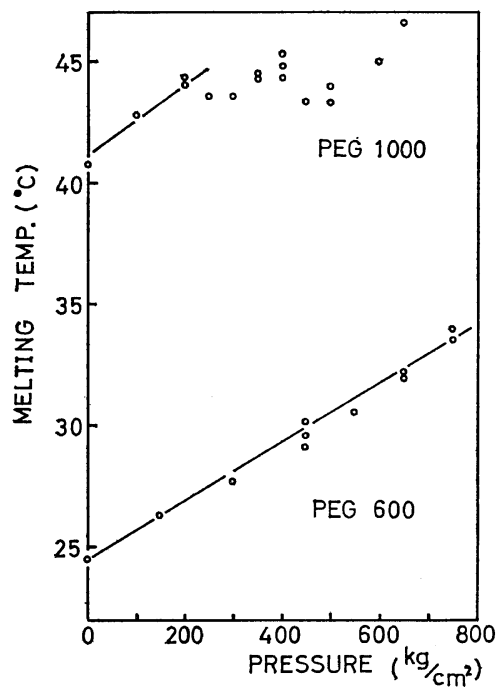

Figure 1. Pressure dependence of melting temperature for PEG 600 and 1000 . melting region. The values of $\Delta V_{\mathrm{m}}$ for PEG and PTHF of $100 \%$ crystallinity were computed by dividing these values by the degrees of crystallinity, and are given in Table I. Melting temperatures at elevated pressures were obtained from measurements of the $V-T$ relation at constant pressure, and they are shown for PEG 600 and PEG 1000 in Figure 1, for PEG 6000 in Figure 2, and for PTHF in Figure 3. The normal increase in $T_{\mathrm{m}}$ with pressure was not observed for PEG 1000 even at somewhat lower pressures. The cause for this is not clear at present. Since the abnormal phenomena, however, do not occur near atmospheric pressure, as seen in Figure 1, the value of $\mathrm{d} T_{\mathrm{m}} / \mathrm{d} P$ at atmospheric pressure, which is required for the

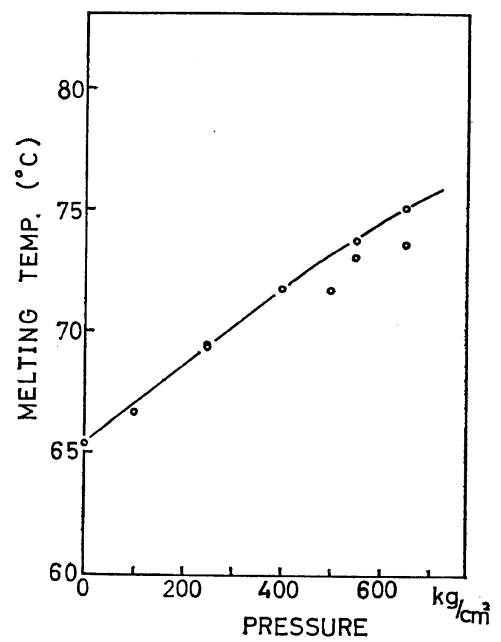

Figure 2. Pressure dependence of melting temperature for PEG 6000 . 
Thermodynamic Properties of Polyethers

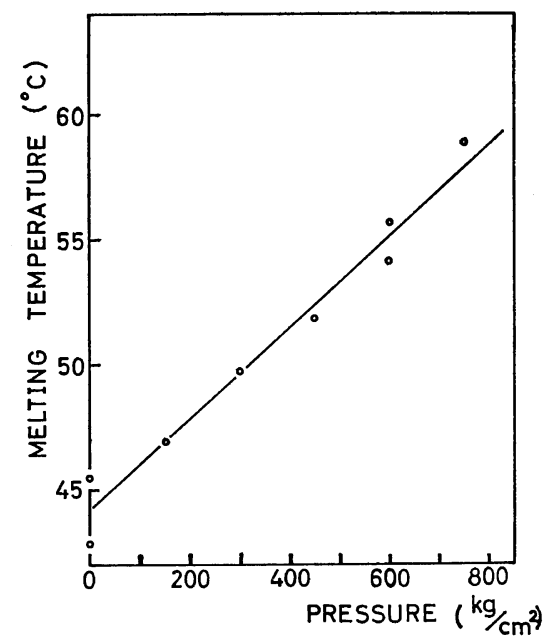

Figure 3. Pressure dependence of melting temperature for poly(tetrahydrofuran).

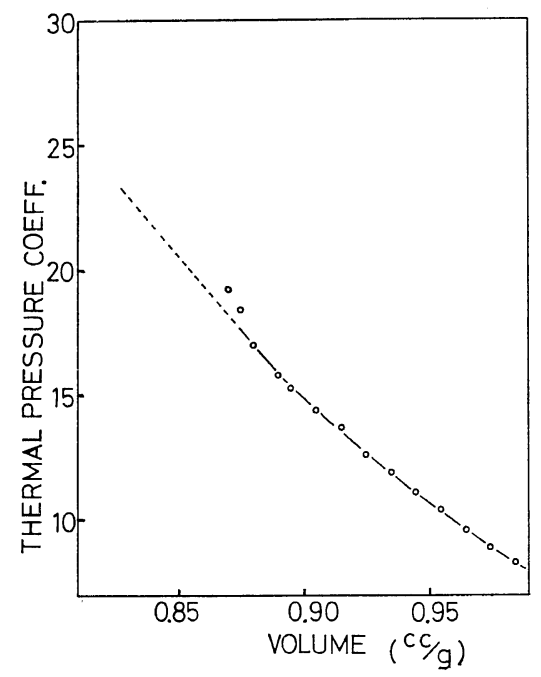

Figure 4. Correlation between thermal pressure coefficient and specific volume for PEG 600 .

calculation of $\Delta S_{\mathrm{m}}$, can be determined. The values of such $\mathrm{d} T_{\mathrm{m}} / \mathrm{d} P$ for all samples are shown in Table I. From the $P-V-T$ relations, ${ }^{3}$ thermal pressure coefficients $\gamma=(\partial P / \partial T)_{V}$ were obtained. The results are shown as functions of specific volume for PEG 600 in Figure 4 and for PTHF in Figure 5.

\section{ESTIMATION OF ENTROPY OF MELTING AND ITS COMPONENTS}

The entropy of melting $\Delta S_{\mathrm{m}}$ is divided into

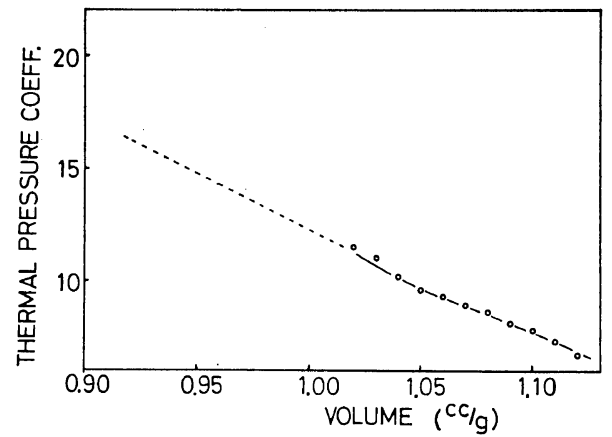

Figure 5. Correlation between thermal pressure coefficient and specific volume for poly(tetrahydrofuran).

three parts, i.e.,

$$
\begin{aligned}
\Delta S_{\mathrm{m}} & =\left(\Delta S_{\mathrm{m}}\right)_{V}+\Delta S_{\mathrm{V}} \\
& =\left(\Delta S_{\mathrm{dis}}+\Delta S_{\mathrm{c}}\right)+\Delta S_{\mathrm{V}}
\end{aligned}
$$

where $\left(\Delta S_{\mathrm{m}}\right)_{V}$ is the entropy of melting at constant volume. Since $\Delta S_{\mathrm{m}}$ and $\Delta S_{\mathrm{V}}$ can be experimentally obtained, $\left(\Delta S_{\mathrm{m}}\right)_{V}$ is evaluated from eq 1. If $\Delta S_{\mathrm{dis}}$ is estimated, $\Delta S_{\mathrm{c}}$ is then evaluated as $\left(\Delta S_{\mathrm{m}}\right)_{V}-\Delta S_{\text {dis }}$.

\section{Entropy of Melting}

The entropy of melting was calculated by the Clausius-Clapeyron equation

$$
\frac{\mathrm{d} T_{\mathrm{m}}}{\mathrm{d} P}=\frac{\Delta V_{\mathrm{m}}}{\Delta S_{\mathrm{m}}}
$$

With substitution of the data for $\Delta V_{\mathrm{m}}$ and $\mathrm{d} T_{\mathrm{m}} / \mathrm{d} P$ into eq 3 , the molar entropies of melting per repeat unit were evaluated, and they are shown in Table I.

\section{Entropy Change Due to Volume Change}

The entropy change due to a change in volume can be evaluated by use of the Maxwell relation

$$
\left(\frac{\partial S}{\partial V}\right)_{T}=\left(\frac{\partial P}{\partial T}\right)_{V}
$$

or

$$
\Delta S_{\mathrm{V}}=\int\left(\frac{\partial P}{\partial T}\right)_{V} \mathrm{~d} V=\int \gamma \mathrm{d} V
$$

The thermal pressure coefficient, $\gamma$, varies with volume as shown in Figures 4 and 5 . Hence it is necessary to take this dependence of $\gamma$ into account in the calculation of $\Delta S_{\mathrm{V}}$. As discussed in previous papers, ${ }^{3,4}$ the internal pressure $P_{\mathrm{i}}$, 
which is approximately equal to $T_{\gamma}$ near atmospheric pressure, increases continuously as the volume decreases in the liquid state. Therefore $\gamma$ may also increase with the decrease in volume, although the volume dependence of $\gamma$ cannot be exactly estimated for a wide range of volume. Hence it is assumed here that $\gamma$ increases with volume at the same slope as in the vicinity of the melting point in the liquid state. This assumption is indicated by the dotted lines in Figures 4 and 5 . The values of $\Delta S_{\mathrm{V}}$ thus obtained by eq 5 are shown in Table I.

\section{Disordering Entropy $\Delta S_{\mathrm{dis}}$}

The disordering entropy was estimated by the same method as in a preceding paper. ${ }^{1}$ Namely, it was assumed that the molar disordering entropy of melting is independent of the particular substance, even for polymers, and is $0.95 \mathrm{cal} /$ mol deg. This value was derived from the experimental data for $\mathrm{Ar}$ and $\mathrm{CCl}_{4}$ with the equation $\Delta S_{\mathrm{dis}}=\Delta S_{\mathrm{m}}-\Delta S_{\mathrm{V}}$, and the assumption that $\Delta S_{\mathrm{c}}=0$ for such simple spherical molecules. ${ }^{1}$ In the case of polymeric substances, we must confront another problem concerning the choice of mole unit. This unit is considered to be the one which has the same external degrees of freedom as simple spherical molecules, i.e., three external degrees of freedom. The size of such a unit is estimated on the basis of a corresponding state theorem to be 1.90 times and 1.27 times the repeating unit for PEG 6000 and PTHF, respectively (see Appendix). The resulting disordering entropies are shown in Table I.

\section{Conformational Entropy $\Delta S_{\mathrm{c}}$}

By subtracting $\Delta S_{\mathrm{dis}}$ from the configurational entropy $\left(\Delta S_{\mathrm{m}}\right)_{V}$, the conformational entropy $\Delta S_{\mathrm{c}}$ is obtained and is given in Table $I$.

Other thermodynamic quantities related to melting are also shown in Table I.

\section{DISCUSSION}

The entropy of melting for PEG decreases with increasing molecular weight. The entropy of melting in PTHF lies between those of poly (ethylene glycol) and polyethylene when compared in the range of high molecular weight. The heat of fusion $\Delta H_{\mathrm{m}}$, evaluated from the thermodynamic equation $\Delta H_{\mathrm{m}}=T_{\mathrm{m}} \Delta S_{\mathrm{m}}$, agrees well with the $\Delta H_{\mathrm{m}}(2.17 \mathrm{kcal} / \mathrm{mol})$ obtained from differential scanning calorimetry. Mandelkern ${ }^{5}$ has obtained the heat of fusion from an analysis of the melting point depression caused by the addition of diluents. His value $\Delta H_{\mathrm{m}}(1.98 \mathrm{kcal} /$ mol) is a little smaller than ours. The heat of fusion increases with increasing molecular weight (see Table I).

Malcolm and Ritchie ${ }^{6}$ reported that the entropy of melting at constant volume for poly(ethylene glycol) with $M=5000$ is $4.22 \mathrm{cal} / \mathrm{mol} \mathrm{deg}$ if the thermal pressure coefficient is assumed to be independent of volume.

Malcolm and Ritchie ${ }^{6}$, theoretically calculated the conformational entropy for PEG to be 3.35 $\mathrm{cal} / \mathrm{moldeg}$, assuming that the difference in potential energy between the trans and gauche forms of the $\mathrm{C}-\mathrm{C}$ bonds in PEG is the same as in polyethylene and that the difference for the $\mathrm{C}-\mathrm{O}$ bonds in PEG is the same as in poly(ethyleneoxide). On the other hand, Tonelli ${ }^{7}$ evaluated the intramolecular configurational entropy on melting for a single isolated polymer chain on the basis of the rotational isomeric state model, and obtained $5.10 \mathrm{cal} / \mathrm{mol} \mathrm{deg}$ for poly(ethylene glycol). Our data for PEG fall between the values of these two authors. The ratios of $\Delta S_{\mathrm{e}}$ to $\Delta S_{\mathrm{m}}$ are $0.66-0.69$ for both PEG and PTHF, whereas those for eicosane and polyethylene are $0.60-0.62$. Thus the contribution of the conformational entropies of polyethers to the total entropy of melting is larger than that of polyethylene.

The conformational entropy $\Delta S_{\mathrm{c}}$ tends to decrease as the molecular weight increases. These results for molecular weight dependence agree qualitatively with those derived from both Smith's equation ${ }^{8}$ and Broadhurst's empirical equation $^{9}$ for $n$-hydrocarbons. The relation between conformational entropy and molecular weight for poly(ethylene glycol) coincides with those for eicosane and polyethylene.

\section{APPENDIX}

The corresponding state theorem is represented by the equation of state

$$
f(\widetilde{P}, \tilde{V}, \tilde{T})=0
$$

where $f$ is a universal function, and $\widetilde{P}=P / P^{*}$, 
$\tilde{V}=V / V^{*}$, and $\tilde{T}=T / T^{*}$ are the reduced pressure, volume, and temperature, respectively. Characteristic pressure, volume, and temperature can be written ${ }^{10}$

$P^{*}=e^{*} / v^{*}, \quad V^{*}=v^{*} N, \quad$ and $T^{*}=e^{*} / c k$

where $e^{*}$ is the intersegmental interaction parameter, $v^{*}$ is the occupied volume of a segment, $3 c$ is the number of external degrees of freedom per segment, and $N$ is the total number of segments in the system. If eq A-1 and eq A-2 are combined, one can obtain the relation

$$
P_{\mathrm{i}} \alpha V_{\mathrm{s}} / R=(c / M) \widetilde{P}_{\mathrm{i}} \tilde{\alpha} \tilde{V}
$$

where $P_{\mathrm{i}}$ is the internal pressure, $\alpha$ is the thermal expansion coefficient, $V_{\mathrm{s}}$ is the specific volume, $R$ is the gas constant, $\widetilde{P}_{1}$ is the reduced value of $P_{\mathrm{i}}$, i.e., $\widetilde{P}_{\mathrm{i}}=P_{\mathrm{i}} / P^{*}$, and $\tilde{\alpha}$ is the reduced value of $\alpha$, i.e., $\tilde{\alpha}=\alpha T^{*}$. The quantity $M / c$ represents the molecular weight of a unit having three external degrees of freedom. Since the value of $\widetilde{P}_{\mathrm{i}} \tilde{\alpha} \tilde{V}$ is the same for all substances at a corresponding state, $M / c$ can be determined from eq A-3 by comparing the value of $P_{\mathrm{i}} \alpha V_{\mathrm{s}} / R$ at the corresponding state with that of a reference substance for which the value of $M / c$ is known. It is assumed here that the melting point is a corresponding state. Since $c$ is considered to be unity for $\mathrm{Ar}, \widetilde{P}_{\mathrm{i}} \tilde{\alpha} \tilde{V}$ at the melting point is evaluated from the value of $P_{\mathrm{i}} \alpha V_{\mathrm{s}} / R$ of $\mathrm{Ar}$ at the melting point. Then, the values of $M / c$ for other substances can be calculated from the values of $P_{\mathrm{i}} \alpha V_{\mathrm{s}} / R$ at their melting points. For example, the values of $P_{\mathrm{i}} \alpha V_{\mathrm{s}}$ for PEG 6000 and PTHF are estimated to be 0.060 and 0.055 cal/g deg from $P-V-T$ measurements. ${ }^{3}$ Thus the units of PEG 6000 and PTHF having three external degrees of freedom are 1.90 times and 1.27 times as large as the respective repeating units.

\section{REFERENCES}

1. Y. Tsujita, T. Nose, and T. Hata, Polymer J., 3, 587 (1972).

2. H. W. Starkweather and R. H. Boyd, J. Phys. Chem., 64, 410 (1960).

3. Y. Tsujita, T. Nose, and T. Hata, Polymer J., 5, 201 (1973).

4. Y. Tsujita, T. Nose, and T. Hata, ibid., 3, 581 (1972).

5. L. Mandelkern, J. Appl. Phys., 26, 443 (1955).

6. G. N. Malcolm and G. L. D. Ritchie, J. Phys. Chem., 66, 852 (1962).

7. A. E. Tonelli, J. Chem. Phys., 52, 4749 (1970).

8. R. P. Smith, J. Polym. Sci., Part A-2, 4, 869 (1962).

9. M. G. Broadhurst, J. Chem. Phys., 36, 2578 (1962).

10. I. Prigogine, "The Molecular Theory of Solutions," North-Holland Publishing Co., Amsterdam, 1957. 